\title{
Origin of the spatial variation of the pairing gap in Bi-based high temperature cuprate superconductors
}

\author{
Michiyasu Mori, ${ }^{1}$ Giniyat Khaliullin, ${ }^{2}$ Takami Tohyama, ${ }^{3}$ and Sadamichi Maekawa ${ }^{1,4}$ \\ ${ }^{1}$ Institute for Materials Research, Tohoku University, Sendai 980-857r, Japan \\ ${ }^{2}$ Max-Planck-Institut für Festkörperforschung, Heisenbergstrasse 1, D-70569 Stuttgart, Germany \\ ${ }^{3}$ Yukawa Institute for Theoretical Physics, Kyoto University, Kyoto 606-8502, Japan \\ ${ }^{4}$ CREST, Japan Science and Technology Agency, Kawaguchi 433-0012, Japan
}

(Dated: October 25, 2018)

\begin{abstract}
Recently, scanning tunneling microscopy on Bi-2212 cuprate superconductor has revealed a spatial variation of the energy gap that is directly correlated with a modulation of the apical oxygen position. We identify two mechanisms by which out-of-plane oxygens can modulate the pairing interaction within the $\mathrm{CuO}_{2}$ layer: a covalency between the $x^{2}-y^{2}$ band and apical $p$-orbital, and a screening of correlation $U$ by apical oxygen polarization. Both effects strongly depend on the apical oxygen position and their cooperative action explains the experiment.

PACS numbers: 74.72.Hs, 74.62.Bf, 74.20.-z, 75.30.Et
\end{abstract}

Various types of high- $T_{\mathrm{c}}$ cuprates have been discovered for the last few decades. As a function of density of charge carriers doped into the $\mathrm{CuO}_{2}$ planes, a superconducting critical temperature $T_{\mathrm{c}}$ shows in general a maximum which varies strongly from one family of cuprates to another. For hole-doped cuprates, a correlation between the maximum $T_{\mathrm{c}}$ and the energy-level separation of in-plane oxygens $\left(\mathrm{O}_{\mathrm{P}}\right)$ and apical ones $\left(\mathrm{O}_{\mathrm{A}}\right)$ has been noticed early on 1, 2]. Hence, a role of apical oxygen on $T_{\mathrm{C}}$ is of considerable interest [1, 2, 3, 4, 4, 5, 6, 7].

In $\mathrm{Bi}_{2} \mathrm{Sr}_{2} \mathrm{CaCu}_{2} \mathrm{O}_{8}$ (Bi-2212), a mismatch between the rock-salt $\mathrm{BiO}_{2}$ layers and the $\mathrm{CuO}_{2}$ planes causes an extra modulation of the crystal structure with a period about $26 \AA$. In such a "supermodulated" lattice, the distance $d$ from the $\mathrm{CuO}_{2}$ layer to the apical oxygen $\mathrm{O}_{\mathrm{A}}$ is periodically varied within the range of $\sim \pm 6 \%$ [8]. Given that bonds within the $\mathrm{CuO}_{2}$ plane itself are much less affected by supermodulation, Bi-2212 material provides a unique opportunity to study the impact of apical oxygen on superconductivity, by monitoring local electronic properties as a function of $d$ that varies spatially within a supermodulation period. This is precisely what is done in the recent scanning tunneling microscopy (STM) experiment by Slezak et al. 9, 10] (see also Ref. 11).

Slezak et al. measured the gap $\Delta$ in a local density of electronic states, and found nearly $10 \%$ spatial variation of $\Delta$ with the same periodicity as supermodulation. They have emphasized that the gap variation is anticorrelated with the $\mathrm{Cu}-\mathrm{O}_{\mathrm{A}}$ distance variation, $\delta \Delta \propto-\delta d$, i.e., the gap increases when $\mathrm{O}_{\mathrm{A}}$ gets closer to the $\mathrm{CuO}_{2}$ plane and vice versa.

This remarkable observation has already been addressed in several papers by introducing variations of coupling constants on a phenomenological level [12, 13]. However, the underlying microscopic mechanism that links the strength of pairing interactions within $\mathrm{CuO}_{2}$ planes with the position of out-of-plane $\mathrm{O}_{\mathrm{A}}$ remains elusive. In this Letter, we discuss the physical origin of high sensitivity of the pairing gap to the $\mathrm{Cu}-\mathrm{O}_{\mathrm{A}}$ distance and explain the anticorrelation effect $\delta \Delta \propto-\delta d$ observed.

Quite in general, the structural shift of apical oxygen may influence the energy gap either via the hopping parameters (hence density of states on the Fermi level), or via the strength of the pairing interaction. In particular, there is a well-known relation between the next-nearestneighbor hopping $t^{\prime}$ and $T_{\mathrm{c}}$, based on the band structure calculations by Pavarini et al. [4]. One has to notice, however, that this observation concerns a comparison between different families of cuprates with different lattice structure. In fact, Pavarini et al. predicted that the variation of the $\mathrm{Cu}-\mathrm{O}_{\mathrm{A}}$ distance within a given compound hardly affects the hopping parameters [14]. Therefore, we focus here on possible electronic mechanisms by which apical oxygens may affect the strength of the pairing potential within the $x^{2}-y^{2}$ band [15].

We find two different ways how the apical oxygen may enter the game. First, a hybridization of "useful" $x^{2}-y^{2}$ band with the "pairing-inert" orbitals of apical oxygens reduces the pairing interaction. Such a destructive effect of covalency is controlled by a relative energy separation between the orbital levels that depends on $\mathrm{Cu}-\mathrm{O}_{\mathrm{A}}$ distance. We illustrate this by an explicit calculation of the Madelung potential as functions of $d$. Second, we show that the superexchange interaction $J$, which is believed to be essential for magnetic correlations and possibly for superconductivity, is very sensitive to the apical $\mathrm{O}_{\mathrm{A}}$ position. This is due to the high polarizability of $\mathrm{O}^{2-}$ anion which has the effect of screening and reducing the energy $U$ needed to move an electron from one ion to another [16, 17]. In Bi-2212, the screening effect and hence the strength of magnetic correlations $J \propto 1 / U$ are spatially modulated because the closer the apical $\mathrm{O}_{\mathrm{A}}$ is, the stronger the screening is. Remarkably, we find that the above two effects, covalency and screening, both favor an anti-phase relation between $\Delta$ and $d$ variations.

Covalency.- We address this effect in terms of the fol- 
lowing Hamiltonian:

$$
\begin{aligned}
H & =\sum_{k \sigma} \epsilon_{k} c_{k \sigma}^{\dagger} c_{k \sigma}-\sum_{k} \Delta\left(\gamma_{k}^{d} c_{k \uparrow}^{\dagger} c_{-k \downarrow}^{\dagger}+\text { h.c. }\right) \\
& +\sum_{k \sigma} \epsilon_{\mathrm{A}} a_{k \sigma}^{\dagger} a_{k \sigma}+\sum_{k \sigma} \nu_{k}\left(a_{k \sigma}^{\dagger} c_{k \sigma}+\text { h.c. }\right) .
\end{aligned}
$$

Here, the first term corresponds to the in-plane $p d \sigma$-band of $x^{2}-y^{2}$ symmetry made of $\mathrm{Cu}$ and $\mathrm{O}_{\mathrm{P}}$ orbitals, and a conventional form of dispersion [4] $\epsilon_{k}=-2 t\left(\cos k_{x}+\right.$ $\left.\cos k_{y}\right)+4 t^{\prime} \cos k_{x} \cos k_{y}-2 t^{\prime \prime}\left(\cos 2 k_{x}+\cos 2 k_{y}\right)-\mu$, where $\mu$ is the chemical potential, is adopted. Second term shows that this band is supposed to host a superconductivity of $d$-wave symmetry, with the gap function $\gamma_{k}^{d}=\left(\cos k_{x}-\cos k_{y}\right) / 2$ and the gap magnitude $\Delta=g \sum_{k} \gamma_{k}^{d}\left\langle c_{-k \downarrow} c_{k \uparrow}\right\rangle$, determined by the strength $g$ of a pairing potential (whose origin is not specified).

Third term in Eq. (11) represents holes on the $2 p_{z}$ orbital of apical $\mathrm{O}_{\mathrm{A}}$ with energy $\epsilon_{\mathrm{A}}$. While a complete model may consider the rich internal structure of the "axial" orbital by Pavarini et al. including $3 d_{3 z^{2}-r^{2}}$ and $4 s$ states of $\mathrm{Cu}$ [4], we consider here a minimal model that captures the essential effects of the axial orbitals. Finally, the last term accounts for a covalent mixing of the $x^{2}-y^{2}$ and $p_{z}$ orbitals. $d$-wave symmetry of the matrix element $\nu_{k}=4 \nu \gamma_{k}^{d}$ is imposed by the hopping geometry: a transfer integral $\nu$ from $x^{2}-y^{2}$ type Zhang-Rice orbital to the neighboring $p_{z}$ states of axial symmetry must have different signs along $x$ and $y$ directions.

Physically, we associate $\epsilon_{\mathrm{A}}$ with the energy-level separation of holes residing on in-plane $\mathrm{O}_{\mathrm{P}}$ and apical $\mathrm{O}_{\mathrm{A}}$ sites, as shown schematically in Fig. 1(b). A magnitude of $\epsilon_{\mathrm{A}}$ can then be estimated from the Madelung potentials on $\mathrm{O}_{\mathrm{P}}$ and $\mathrm{O}_{\mathrm{A}}$ [18] using the structural data of Ref. 8 . To calculate variations of $\epsilon_{\mathrm{A}}$ caused by supermodulation, we use the displacement pattern, Fig. 1(a), inferred from the structural data. This way, we quantify $\epsilon_{\mathrm{A}}$ in terms of the distance $d$ from the top $\mathrm{CuO}_{2}$ layer (relevant for STM) to $\mathrm{O}_{\mathrm{A}}$ above it, making thereby a link between the model and supermodulation.

Below, we use a representative hopping parameters $t=0.4 \mathrm{eV}, t^{\prime} / t=0.3, t^{\prime \prime}=t^{\prime} / 2$, and $\nu / t=0.35$. The $d-$ wave momentum dependence of $\nu_{k}$ renormalizes the $t^{\prime}, t^{\prime \prime}$ values but we compensate this numerically by adding a counter-term $\propto \nu^{2} / \epsilon_{\mathrm{A}}$ and keep the actual values of $t^{\prime}, t^{\prime \prime}$ invariant against the $\mathrm{O}_{\mathrm{A}}$ shifts (as found in the band structure calculations [4]). The covalency effect is then entirely due to the spectral weight shifts between $x^{2}-y^{2}$ and axial orbitals.

Calculating the expectation value $\left\langle c_{-k \downarrow} c_{k \uparrow}\right\rangle$ in the model (1), we find the following gap equation:

$$
1=g \sum_{k} \sum_{ \pm} \frac{Z_{ \pm}}{2 E_{ \pm}} \tanh \frac{E_{ \pm}}{2 T}\left|\gamma_{k}^{d}\right|^{2}
$$

The quasiparticle energies are given by $E_{ \pm}=\frac{1}{\sqrt{2}}\left[\epsilon_{k}^{2}+\right.$ $\left.\Delta_{k}^{2}+\epsilon_{\mathrm{A}}^{2}+2 \nu_{k}^{2} \pm R^{2}\right]^{1 / 2}$, where $R^{2}=\left\{\left(\epsilon_{\mathrm{A}}^{2}-\epsilon_{k}^{2}-\Delta_{k}^{2}\right)^{2}+\right.$
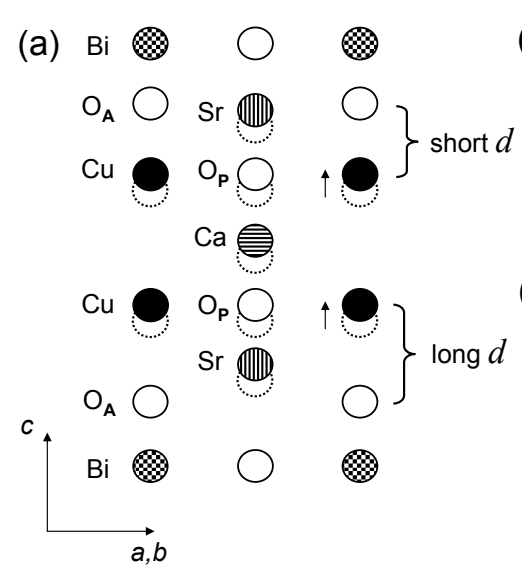

(b)

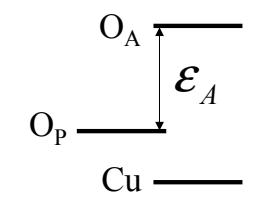

(c)

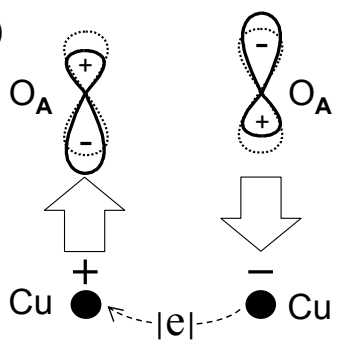

FIG. 1: (a) Local atomic displacements along the $c$ axis due to the supermodulation (exaggerated). The $\mathrm{Bi}_{-} \mathrm{O}_{\mathrm{A}}$ bond length does not change much but other atoms $\left(\mathrm{Cu}, \mathrm{O}_{\mathrm{P}}, \mathrm{Sr}\right.$, and $\mathrm{Ca}$ ) are shifted such that the $\mathrm{Cu}-\mathrm{O}_{\mathrm{A}}$ bond is most affected [8]. The direction (up/down) and amplitude of the shift $\delta d$ modulates along the $a$ axis with a period $\sim 26 \AA$, resulting in a $\delta d \sim \pm 6 \%$ variation of the $\mathrm{Cu}-\mathrm{O}_{\mathrm{A}}$ bond length [8]. (b) Energy level scheme of $\mathrm{Cu}, \mathrm{O}_{\mathrm{P}}$, and $\mathrm{O}_{\mathrm{A}}$. (c) Schematic picture of the screening effect: Once a charge is moved from one $\mathrm{Cu}$-ion to another (at the energy cost $U$, initially), the apical oxygen orbitals experience an electric field (arrows) of excited charges and are polarized. An energy gain from the polarization process reduces a virtual charge excitation energy $U$.

$\left.4 \nu_{k}^{2}\left[\left(\epsilon_{k}+\epsilon_{\mathrm{A}}\right)^{2}+\Delta_{k}^{2}\right]\right\}^{1 / 2}$. Eq. (2) is composed of two parts where $Z_{ \pm}=\left(E_{ \pm}^{2}-\epsilon_{\mathrm{A}}^{2}\right) /\left(E_{ \pm}^{2}-E_{\mp}^{2}\right)$ represent the spectral weights of the pairing-active $x^{2}-y^{2}$ orbital on the two bands $E_{ \pm}$. We note that $Z_{-}$(which is the most relevant one) is reduced from its bare value $(=1$ at $\nu=0)$ due to the orbital mixing. This has the effect of reducing effective value of $g$ in Eq. (2).

First, we regard $\epsilon_{A}$ as a free parameter and consider how its variation affects the gap. Solving Eq. (2) at $g / 4 t=0.9$ and $T=0$, we obtained a sizable variation of the gap as a function of $\epsilon_{A}$ as shown in Fig. 2(a). This is due to the covalency effect that reduces the spectral weight of the $x^{2}-y^{2}$ states near the Fermi-level, by transferring it to the higher energy apical states. An amount of hole transferred into the apical level is rather small, of the order of several percent, see Fig. 2(b). However, it may be observed in the nuclear quadrupole resonance (NQR) which is sensitive to the hole concentration [22].

Next, we consider how $\epsilon_{\mathrm{A}}$ is shifted by the structural modulation. From the Madelung potential calculation, we obtained a linear relation $\epsilon_{\mathrm{A}}(d) / t=\left(\bar{\epsilon}_{\mathrm{A}} / t\right)[1-$ $\left.a\left(d / d_{0}-1\right)\right]$, with $\bar{\epsilon}_{\mathrm{A}} / t=3.85[20]$ and $a=1.6 . d_{0} \simeq 2.4$ $\AA$ is an average $\mathrm{Cu}-\mathrm{O}_{\mathrm{A}}$ distance [8]. The inset of Fig. 2(a) shows that the energy level separation $\epsilon_{\mathrm{A}}$ increases as the apical site $\mathrm{O}_{\mathrm{A}}$ comes closer to the $\mathrm{Cu}$-ion. Consequently, the pairing gap is also increased [see Fig. 2(a)], since the strength of the hybridization is reduced. 

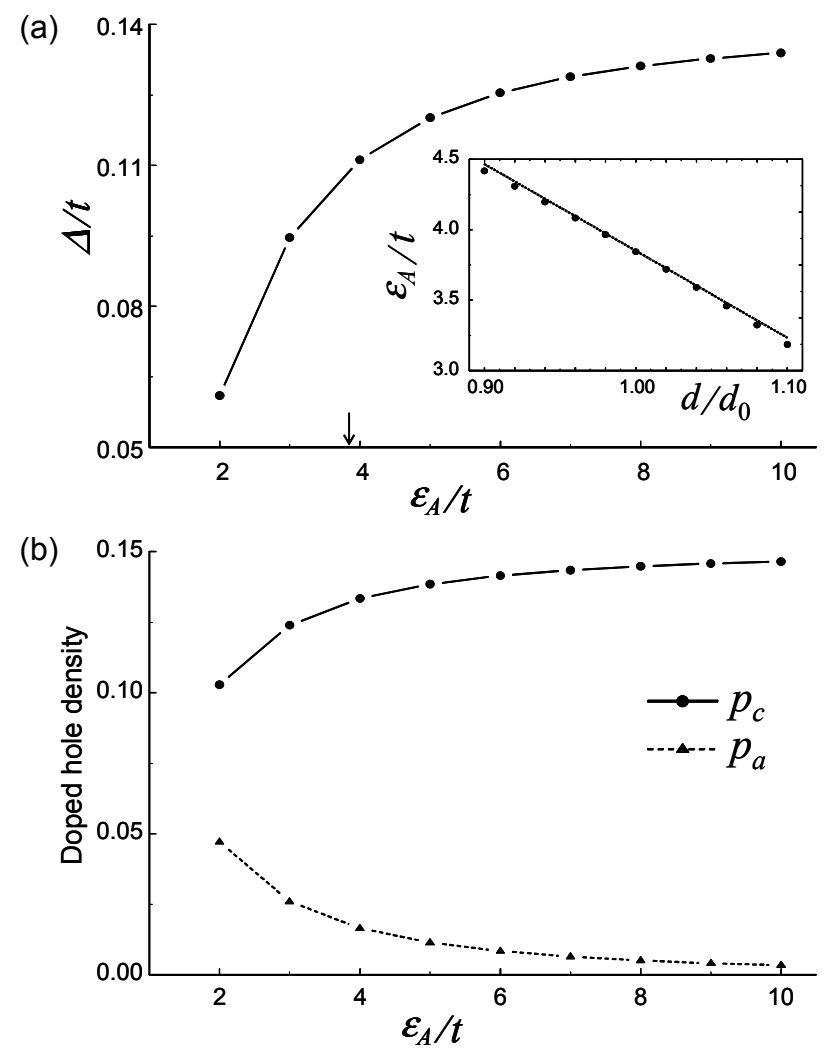

FIG. 2: (a) The pairing gap $\Delta$ as a function of the energylevel separation $\epsilon_{\mathrm{A}}$ between the apical orbital $a$ and the $x^{2}$ $y^{2}$ band. Arrow indicates $\epsilon_{\mathrm{A}}$ at $d=d_{0}$. Inset: $\epsilon_{\mathrm{A}}$ vs. $\mathrm{Cu}-\mathrm{O}_{\mathrm{A}}$ distance $d$. The data are fitted by a linear relation $\epsilon_{\mathrm{A}}(d) / t=3.85\left[1-1.6\left(d / d_{0}-1\right)\right]$. (b) Distribution of doped holes among the $x^{2}-y^{2}$ and the $a$ bands, denoted by $p_{\mathrm{c}}$ and $p_{\mathrm{a}}$, respectively. Total density of holes is fixed to $p_{\mathrm{c}}+p_{\mathrm{a}}=0.15$.

Having obtained the relations $\Delta$ vs $\epsilon_{\mathrm{A}}$ and $\epsilon_{\mathrm{A}}$ vs $d$, we are now in position to show the gap $\Delta$ variations as a function of $d$ directly. The result is presented in Fig. 3 by the broken line, which shows that the $\pm 6 \%$ change of $d$ leads to a sizable variation of $\Delta$.

Screening.- This effect is based on an observation 16, 17] that anion polarization renormalizes the energy of virtual charge excitations. This physics is relevant here since the $\mathrm{O}_{\mathrm{A}}$-contribution to the screening of in-plane interactions should strongly depend on $d$.

Let us consider how $U$ and magnetic correlations within the $\mathrm{CuO}_{2}$ planes are modified by apical $\mathrm{O}_{\mathrm{A}}$. In the $U$-excited intermediate state, both an unoccupied and doubly occupied $\mathrm{Cu}$ sites strongly polarize apical sites just above each $\mathrm{Cu}$ site, see Fig. 1(c). Apical oxygen obtains a dipole moment $p=\alpha F$ where $\alpha$ is the polarizability of $\mathrm{O}^{-2}$ ion, and $F \simeq \mathrm{e} / d^{2}$ is an electric field on oxygen induced by an extra charge (hole or electron) on $\mathrm{Cu}$-site which is created by $U$-excitation. (We ignored dipolefield corrections to $F$ from further located ions for simplicity). Energy gain due to this polarization process in a virtual state reduces the $U$-excitation energy to $U_{\text {eff }}(d)=$

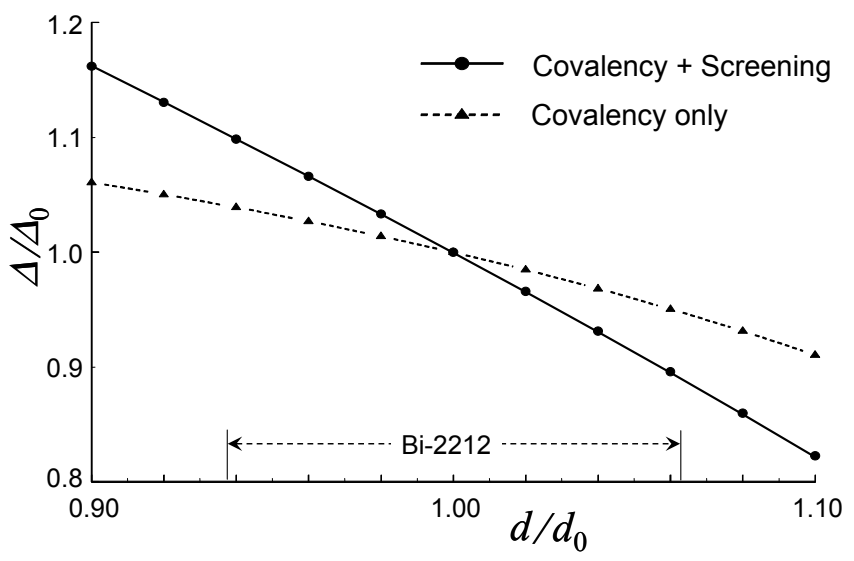

FIG. 3: The gap $\Delta$ as a function of $d$, where $\Delta_{0} \equiv \Delta\left(d_{0}\right)$. The broken line shows the covalency effect only, i.e., the gap variation is solely due to modulation of $\epsilon_{\mathrm{A}}(d)$ at fixed $g / 4 t=$ 0.9. The solid line includes also the screening effect through the $d$-dependence of the coupling constant $g(d)$ (see text). In $\mathrm{Bi}-2212, \mathrm{Cu}-\mathrm{O}_{\mathrm{A}}$ distance varies within the range indicated.

$U-E_{\mathrm{pol}}(d)$, where $E_{\mathrm{pol}}(d)=2(p F / 2)=\alpha F^{2} \simeq \alpha\left(\mathrm{e} / d^{2}\right)^{2}$ is an interaction energy between the induced dipole moments $p$ on $\mathrm{O}_{\mathrm{A}}$ and an excited hole (electron) at the unoccupied (doubly-occupied) $\mathrm{Cu}$-sites. Since $\mathrm{O}^{-2}$ ion has large polarizability, $\alpha \simeq 2 \AA^{3}[23]$, energy $E_{\text {pol }}(d)$ is sizable: for the average $\mathrm{Cu}-\mathrm{O}_{\mathrm{A}}$ distance $d_{0} \simeq 2.4 \AA$ we estimate $E_{0} \equiv E_{\text {pol }}\left(d_{0}\right) \simeq 0.9 \mathrm{eV}$. It is important to realize that, due to the strong $d$-dependence of $E_{\mathrm{pol}}(d) \propto 1 / d^{4}$, the effective repulsion $U_{\text {eff }}$ becomes highly sensitive to the $\mathrm{Cu}-\mathrm{O}_{\mathrm{A}}$ distance: $\delta U_{\text {eff }}(d)=-\delta E_{\mathrm{pol}}(d)=4 E_{0} \delta d / d_{0}$. An immediate consequence of this observation is that the antiferromagnetic exchange interaction $J \simeq 4 t^{2} / U_{\text {eff }}$ between the $\mathrm{Cu}$-spins obtains the same strong modulation as a function of $d: \delta J(d) / J_{0}=-\left(4 E_{0} / U_{0}\right)\left(d / d_{0}-1\right)$, where $U_{0} \equiv U_{\text {eff }}\left(d_{0}\right)$ and $J_{0} \equiv J\left(d_{0}\right)$. With the above estimate for $E_{0}$ and using a representative value $U_{0} \simeq 7$ $\mathrm{eV}$, we find that $\delta J(d) / J_{0}=-\beta\left(d / d_{0}-1\right)$ with $\beta \simeq 1 / 2$. Thus, in a modulated structure of Bi-2212, the $J$ value strongly increases as the $\mathrm{Cu}-\mathrm{O}_{\mathrm{A}}$ distance $d$ decreases and vice versa, i.e., it shows the same anticorrelation effect with $d$ as the pairing gap $\Delta$ does in the experiment.

Now, we assume that the superexchange driven magnetic correlations are essential for the pairing in cuprates as widely believed. Indeed, e.g., in the $t-J$ model, $J$ plays a role of pairing potential same as $g$ in Eq. (2). It is then natural to consider that the pairing potential $g$ in Eq. (2) is modulated in the same functional form as $J$. We therefore implement a relation $g(d)=g\left[1-\beta\left(d / d_{0}-\right.\right.$ 1)] with $\beta=1 / 4$ [24] and calculate the gap values from Eq. (2) for different $d$. The obtained gap modulation is presented in Fig. 3 by the solid line. A combined action of the covalency and the screening effects can be summarized by a relation $\delta \Delta / \Delta_{0} \approx-A \cdot \delta d / d_{0}$, with $A \simeq 1.6$.

For a comparison of this result with experiment [10], we notice that the measured gap $\Delta(\mathbf{r})$ is in fact de- 
termined by a "coarse-grained" value $\tilde{d}(\mathbf{r}) \approx\langle d(\mathbf{r})\rangle_{\xi}$ of the actual $\mathrm{Cu}-\mathrm{O}_{\mathrm{A}}$ distances. Such a coarse-graining of $\delta d \propto \cos \left(2 \pi r_{a} / \lambda\right)[10]$ gives $\delta \tilde{d}(\mathbf{r}) \approx f \cdot \delta d(\mathbf{r})$, i.e., the modulation "seen" by Cooper pairs is reduced by a factor $f$ that depends on the ratio of the coherence length $\xi$ and the supermodulation period $\lambda \approx 26 \AA[25]$. At $\xi \approx 20$ $\AA$, we find $f \approx 0.43$. The above relation $\delta \Delta$ vs $\delta d$ reads then as $\delta \Delta / \Delta_{0} \approx-\tilde{A} \cdot \delta d / d_{0}$, where $\tilde{A} \approx 0.7$. This gives $\approx 9 \%$ total variation in $\Delta$ due to $\pm 6.25 \%$ modulation of $d$, just as observed by Slezak et al..

In a broader context, it should be emphasized that while we are concerned here with the variations of apical oxygen position within a given structure of a given material, the effects discussed - spectral weight transfer, and screening of effective $U$ - are generic and relevant for the gap and $T_{c}$ variations among different cuprate families. However, many other things must be kept in mind when we compare different cuprates. In particular, apical oxygens may have a negative impact on $T_{c}$ by communicating a destructive effect of out-of-plane disorder to the $\mathrm{CuO}_{2}$ planes [5, 6]. Anticorrelation between the energy gap and the distance to the apical oxygen observed by Slezak et al. in Bi-2212 implies that the effects we discussed here overcome the disorder related physics (which would result in a trend opposite to what observed). A key question is then how this competition is resolved in different cuprate families. To address this issue and better understand the $T_{c}$ trends in cuprates, our model has to be implemented by out-of-plane disorder effects.

Finally, we argued that effective $U$ hence $J$ values are renormalized by $\mathrm{O}_{\mathrm{A}}$ and thus they become sensitive to the $\mathrm{O}_{\mathrm{A}}$-position. This inevitably turns the exchange interaction in $\mathrm{Bi}-2212$ into inhomogeneous one in space. In other words, we expect that the strength of local spin correlations follow the lattice supermodulation. The resulting broad distribution of relaxation times could possibly be tested by the NMR/NQR experiments. In fact, the recent neutron scattering work has revealed an intrinsic broadening of the spin excitations in Bi-2212 26], an observation that seems natural in a light of our picture.

To conclude, we discussed the physical origin of the relationship between the pairing energy gap and the atomic displacements in the supermodulated structure of $\mathrm{Bi}$ 2212. A covalent mixing of the $x^{2}-y^{2}$ orbital with apical $p$-level, and a screening of effective $U$ values via the polarization of apical oxygens are found to act cooperatively and modulate the pairing correlation as a function of the $\mathrm{Cu}-\mathrm{O}_{\mathrm{A}}$ distance. This leads to the spatial variations of the energy gap as observed in the experiment.

We would like to thank J.C. Davis and B. Keimer for useful discussions. This work was supported by a Grandin-Aid for Scientific Research on Priority Areas and the NAREGI Nanoscience Project from MEXT. M.M. was supported by a Grand-in-Aid for Young Scientists (B). G.Kh. thanks IFCAM at IMR, Tohoku University, and YKIS07, Yukawa International Program at YITP, for kind hospitality. The authors thank the Supercomputer Center, ISSP, University of Tokyo.

[1] Y. Ohta, T. Tohyama, and S. Maekawa, Phys. Rev. B 43, 2968 (1991).

[2] S. Maekawa et al., Physics of Transition Metal Oxides, Springer Series in Solid State Sciences, vol. 144 (SpringerVerlag, Berlin, 2004).

[3] H. Matsukawa and H. Fukuyama, J. Phys. Soc. Jpn. 59, 1723 (1990).

[4] E. Pavarini et al., Phys. Rev. Lett. 87, 047003 (2001).

[5] H. Eisaki et al., Phys. Rev. B 69, 064512 (2004).

[6] Q.Q. Liu et al., Phys. Rev. B 74, 100506 (2006).

[7] K. McElroy et al., Science 309, 1048 (2005).

[8] A. Yamamoto et al., Phys. Rev. B 42, 4228 (1990).

[9] J.A. Slezak, Ph.D. thesis, Cornell University, Ithaca, 2007.

[10] J.A. Slezak et al., Proc. Natl. Acad. Sci. USA 105, 3203 (2008).

[11] M.R. Norman, Proc. Natl. Acad. Sci. USA 105, 3173 (2008).

[12] B.M. Andersen, P.J. Hirschfeld, and J.A. Slezak, Phys. Rev. B 76, 020507(R) (2007).

[13] K.-Y. Yang, T.M. Rice, and F.-C. Zhang, Phys. Rev. B 76, 100501(R) (2007).

[14] See the lines in Fig. 4 of Ref. 4.

[15] Concerning phonons, experiment shows that $B_{1 \mathrm{~g}}$ phonon modes (which may support $d$-wave pairing) are hardly affected by supermodulation [9], and, moreover, their isotope shift has no effect on the gap variations.

[16] D.K.G. de Boer, C. Haas, and G.A. Sawatzky, Phys. Rev. B 29, 4401 (1984).

[17] J. van den Brink et al., Phys. Rev. Lett. 75, 4658 (1995).

[18] The Madelung potentials are calculated in the ionic model, and are further divided by a dielectric constant $\epsilon_{H}=5$ [19] accounting for the screening of ionic potentials on a phenomenological level.

[19] J. Hwang, T. Timusk, and G.D. Gu, J. Phys.: Condens. Matter 19, 125208 (2007).

[20] The result $\bar{\epsilon}_{\mathrm{A}}=3.85 t$ is consistent with the band structure studies [21] which find $\sim 1.5-2.0 \mathrm{eV}$ separation between $\mathrm{O}_{\mathrm{A}}$ and $\mathrm{O}_{\mathrm{P}}$ derived bands.

[21] D.L. Novikov and A.J. Freeman, Physica C 216, 273 (1993); D.J. Singh and W.E. Pickett, ibid. 235-240, 2113 (1994).

[22] G.-q. Zheng, Y. Kitaoka, K. Ishida, and K. Asayama, J. Phys. Soc. Jpn. 64, 2524 (1995).

[23] R.D. Shannon, J. Appl. Phys. 73, 348 (1993).

[24] We have chosen a conservative value $\beta=0.25$ because, apart from the $U$-process, $J$ is contributed also by socalled charge-transfer term $J_{\Delta}=4 t^{2} /\left(\Delta_{p d}+U_{p} / 2\right)$ where the excited state contains two holes on $O_{P}$ with a correlation energy $U_{p}$. Considering the Madelung potentials and screening of the $p d$-transfer energy $\Delta_{p d}$ by apical oxygens in a similar way as for the $U$-process, we find that $J_{\Delta}$ is less modulated than $J_{U}$, i.e. $\beta_{\Delta} \simeq 0.1$. Thus, an estimate $\beta \sim 0.3$ follows from $U \sim \Delta_{p d}+U_{p} / 2$.

[25] Specifically, $f=(2 / x) J_{1}(x)$, where $J_{1}$ is Bessel function and $x=\pi \xi / \lambda . f=1(f=0)$ if $\lambda \gg \xi(\lambda \ll \xi)$.

[26] B. Fauque et al., Phys. Rev. B 76, 214512 (2007). 